\title{
Destruction of peripheral white matter of the spinal cord, brain-stem, and optic tracts ${ }^{1}$
}

\author{
REINHARD L. FRIEDE AND UROS ROESSMANN \\ From the Institute of Pathology, Case Western Reserve University, Cleveland, Ohio, U.S.A.
}

This report concerns a case with unusual lesions consisting of massive destruction of myelinated fibres in a thin marginal layer at the surface of the spinal cord, brain-stem, optic nerve and tract, without involvement of the superficial portions of grey matter. The lesions showed a striking similarity to those produced by barbotage (aspiration and reinjection of cerebrospinal fluid) in experimental animals (Bunge, Bunge, and Ris, 1960). Our case also resembled the experimental model in that it received numerous therapeutic drainings of cerebrospinal fluid because of a cerebrospinal fluid fistula in the sella.

\section{CASE HISTORY}

A 54-year-old white woman consulted a physician approximately one year before death with carcinoma of the right breast, which was found to be inoperable. She was given a course of $x$-ray treatment with intravenous antimetabolites of unknown nature. There was no significant response to the therapy, and wihin six months the patient complained of back pains extending into the left arm and hip. Three months before death she was given a two-week course of irradiation to the lower spine. Because of relentless progression of the tumour at the primary site and obvious metastases to the other breast and skeletal system, she was referred to the University Hospitals for hypophysectomy.

On physical examination she was found to have multiple, hard nodules in both axillae and right supraclavicular areas, obvious mass lesions in both breasts with satellite nodules, diffuse spinal tenderness; there was no neurological deficit.

Shortly after admission an uncomplicated transsphenoidal hypophysectomy was done with apparent complete removal of the pituitary gland. The sella and sphenoid sinus were plugged with two segments of muscle and the patient was said to have tolerated the procedure well. During the post-operative course, the patient received Gantrisin, erythromycin and, later, tetracycline and streptomycin; all were given intravenously or per os. She was on corticoids during the entire course, and thyroxin was given during the last five days.

${ }^{1}$ This investigation was supported by Public Health Grant NB 06239 from the National Institute of Neurological Diseases and Blindness.
Three days after surgery it became evident that a leas had developed at the surgical site and her condition was checked with daily spinal taps and she was treated wi antibiotics. Some 30 to $40 \mathrm{ml}$. of cerebrospinal fluid were removed with each tap, and the patient complained of severe headache. On the ninth post-operative day the surgical wound was revised and closed again with $\overrightarrow{\vec{a}}$ segment of iliac bone, muscle, and Surgicel impregnatefg with Eastman 910. During surgery the sella was irrigate with bacitracin. Lacerations in the nasal mucosa were resutured. Spinal tapping was continued post-operative and for five days no rhinorrhoea was detected; severap cultures of CSF were positive for coliform organisms.

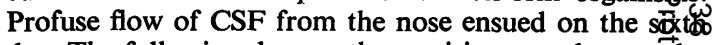
day. The following day another revision was done uffie nitrous oxide anaesthesia. During surgery it was nfeg that she required little anaesthetic, and afterwards ohe was found to have dilated and fixed pupils. She respondeg very slowly but remained obtunded; there were $\mathrm{n} \overline{\mathrm{e}}$ localizing findings and no pathological reflexes. Her bto pressure began to drop and continuous infusion vasopressors was necessary. After the initial improvenaing in sensorium the condition continued to deteriorate; io 24 hours she became completely unresponsive, and aill therapeutic measures were of no avail. She died sis days after the last surgical procedure.

NECROPSY FINDINGS The autopsy was performed thre hours after death. There was widespread carcinoma of the right breast, metastatic to the regional lymph nodes tracheo-bronchial lymph nodes, right pleura, left breas adrenals, bone and skin. Other findings were bronchopneumonia with left lower lobe abscess formation, an a perforated duodenal ulcer. There was no invasion of central nervous tissue or CSF spaces by carcinoma.

The brain, weighing $1,125 \mathrm{~g}$, was fixed by suspensiợ from the basal artery. The spinal cord was removes entirely. Gross inspection of the brain showed a mile purulent leptomeningitis, a recent subdural hematoma \& few millimetres thick, and uncal herniation. The pituitar gland was absent. The floor of the sella showed a fistulas about $5 \mathrm{~mm}$ in diameter, communicating widely throug $\mathrm{F}$ the ethmoid sinus to the nasopharynx. The sella w removed en bloc and decalcified. The surface lesions. were not noticed at the time of brain cutting; in retrospectr they could be distinguished only with difficulty, appearing as a thin greyish zone of tissue at the surface of the corfes and brain-stem. Paraffin sections, $10 \mu$ thick, were staineł⿺ 
with haematoxylin and eosin, Bacsich and Stibbe's (1937) method for myelin, Bodian stains, and Holzer stains. Additional frozen sections were stained with oil red $\mathrm{O}$.

HISTOLOGICAL FINDINGS Sections of spinal cord, brainstem, and optic tracts showed loss of tissue and extreme sponginess in a subpial layer of up to $1 \mathrm{~mm}$ thickness. These changes occurred only where the white matter bordered external cerebrospinal fluid spaces. Myelin and Bodian stains indicated a massive loss of myelin sheaths and nerve fibres from the zone of sponginess, although a few scattered myelinated fibres persisted in many portions of the lesions. The border between the spongy zone and the adjacent white matter was fairly sharp, and no changes were seen deeper in the white matter. Swollen axons were absent in many slides; in others, they were scattered mostly at the border of the spongy layer, or-rarely-within the lesions. Few reactive changes were seen in the glial tissue; swollen astrocytes were notably absent. In Holzer stains there was no increase in glial fibrils throughout the lesions. Fat stains demonstrated minute fat droplets in microglia cells in the lesion, and fat-laden macrophages were seen at random or in perivascular arrangement. The latter varied in density from slide to slide and were absent in some. A mild, acute leptomeningitis was present; the exudate was scattered, with a preferential distribution at the base of the brain and the spinal canal. There were no proliferative changes in the connective tissue of the arachnoid.

In the spinal cord the zone of sponginess covered most of the circumference, with a tendency to be thicker at the ventral than dorsal surface (Fig. 1). The lesion extended across the ventral median fisure without following the surface of the cord into the fissure. Entrance zones of the dorsal and ventral roots within the cord were spared. The roots themselves were mostly without pathological changes, but pallor and evidence of myelin breakdown was found in some of the dorsal roots. A mild pallor of the central portion of Goll's tracts was noted in myelin stains with a corresponding slight gliosis in Holzer preparation, but loss of axons was hardly noticeable in Bodian stains. The grey matter was without changes; in particular, no chromatolytic changes were found in the motor neurones of the anterior horn.

Lesions occurred in the medulla oblongata (Fig. 2), pons and mesencephalon over the entire ventral and lateral surface of the brain-stem; they diminished in thickness dorsally and stopped where grey matter lies at the surface-for example, at the cerebellar cortex (Fig. 3) or at the colliculi. Several recent micro-infarcts were found in the central portion of the pons, showing pallor, eosinophilic degeneration of neurones and early disintegration of myelin without marked glial response.

Longitudinal sections through the entrance zone and the glia-Schwann cell borders of roots were available for the cranial nerves III, V, VI, IX, and XII. The central portions of these roots were generally more severely affected than the peripheral portions. Complete loss of myelin or persistence of scattered myelinated fibres were seen in the central portion; the distal portion of the root showed either diffuse pallor distal to the glia-Schwann cell border or evidence of fragmentation of sheaths in scattered fibres. These changes appeared to subside with the distance from the brain-stem. Swollen axons were seen occasionally in Bodian stains. These changes were most marked in the roots of the Vth nerve, and a few scattered neurones with axonal reaction were found in the trigeminal motor nucleus.

The free portion of the optic nerve and tract showed sponginess over its entire surface; where the tract merged with the hemispheres, changes were found only in its exposed surface, without involvement of the adjacent temporal cortex and tuber cinereum (Fig. 4). No surface lesions were found in the cerebral cortex or cerebellar cortex. However, there was diffuse eosinophilic

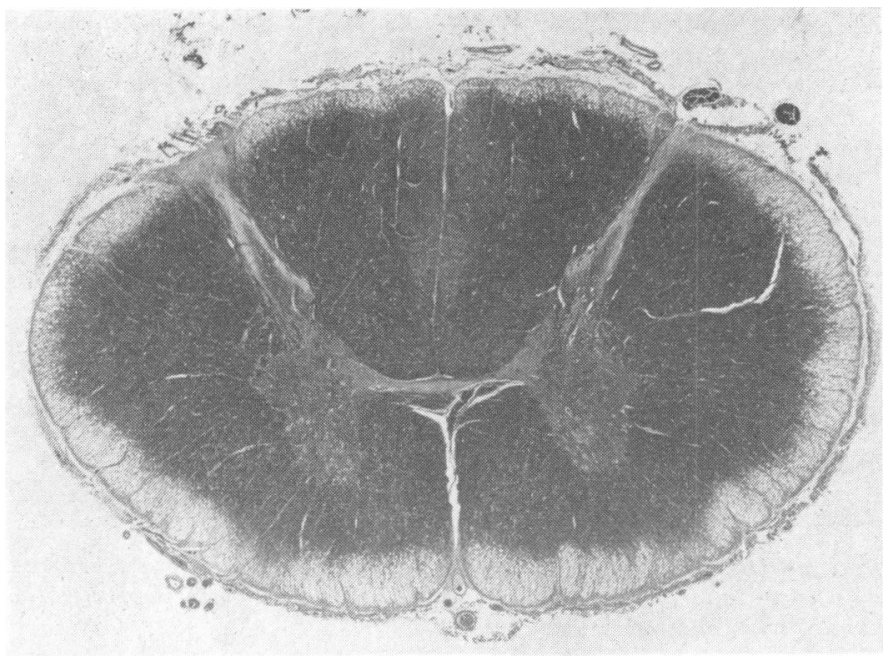

FIG. 1. Massive destruction of white matter at the periphery of spinal cord; minimal pallor of the central portion of Goll's tract. Dorsal and ventral rootlets do not show loss of myelin. Bacsich's stain, $\times 6$. 


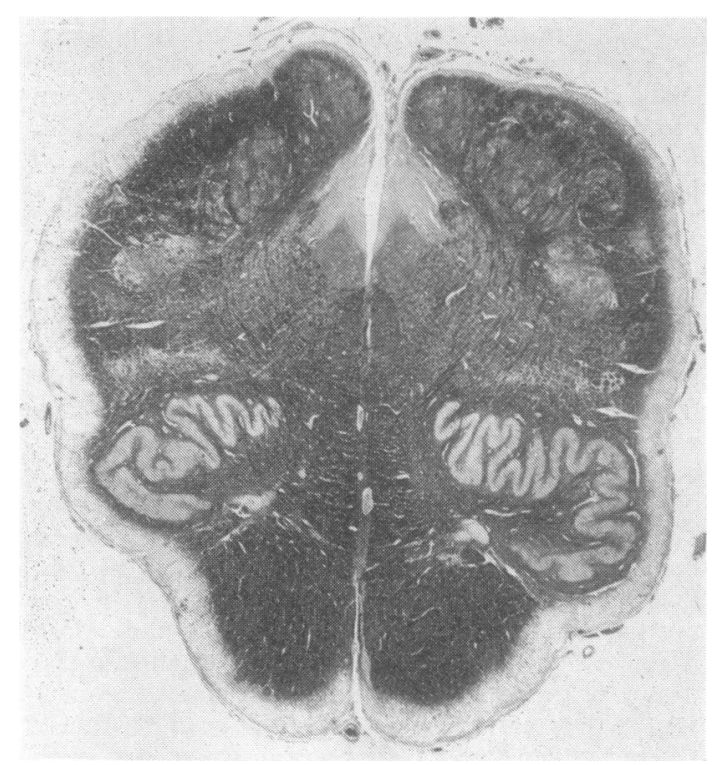

FIG. 2. Peripheral destruction of white matter in the medulla oblongata, decreasing in thickness from ventral to dorsal. Bacsich's stain, $\times 5$.

degeneration and neuronal loss in the temporal cortex, consistent with anoxic encephalopathy, and there were small infarcts in the occipital cortex. There were no lesions at either the dorsal or ventral surface of the corpus callosum. The walls of the cerebral ventricles were free of changes, regardless of whether they were formed by grey or white matter.

Histological sections of the decalcified sella showed cavity with a small portion of surviving anterior an posterior pituitary lobe at its periphery and surgical transplants of necrotic skeletal muscle and bone. sinus tract, lined by necrotic tissue and communicating with sphenoid sinus, was evident in the floor of the sel and showed acute and chronic inflammatory changes in the adjacent tissue.

\section{DISCUSSION}

Several factors must be considered in the pathe genesis of the surface lesions found in this cas $\oplus_{\text {. }}$ Artefacts can be ruled out by the histological findings of neutral fat, lipid-laden macrophages, and swollen axons in the lesions. Improper osmo larity or composition of the fixative would affect the surface of grey and white matter alike; the would also not account for the massive loss of both myelin and axons underneath an intact pia mate? The purulent meningitis found in our case was mild with a scanty exudate, and there were no changes indicating a chronic course. Massive destruction $\&$ the surface of white matter is not a known comple cation of acute purulent leptomeningitis. The oher findings in the CNS of recent and organizing infarets and loss of nerve cells in pons and cerebral corts were consistent with the events preceding the degt

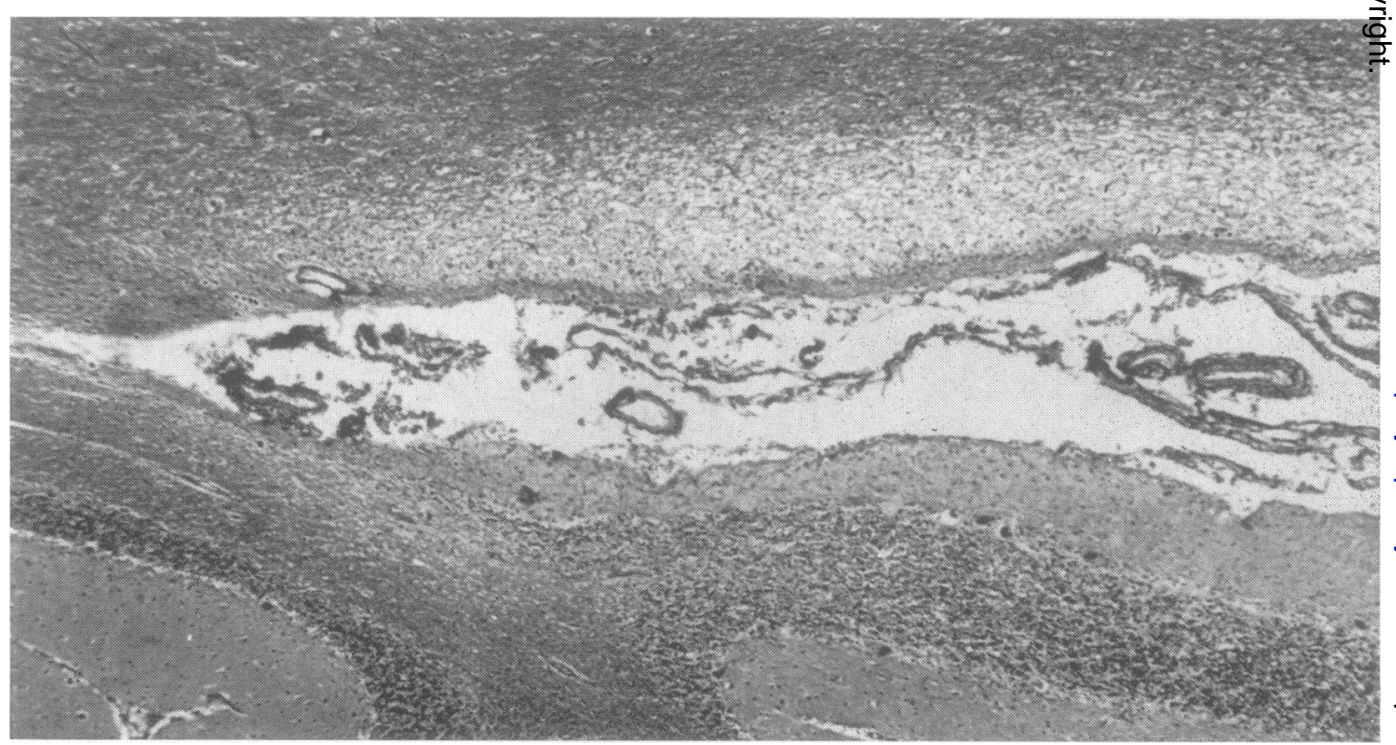

FIG. 3. Superficial destruction of white matter covers the surface of the middle cerebral peduncle, but terminates sharply at the cerebellar cortex. There are no changes in the latter. The section is stained with Bodian'sstain, demonstrat ing the massive loss of axons from the affected portions of white matter. $\times 08$. 


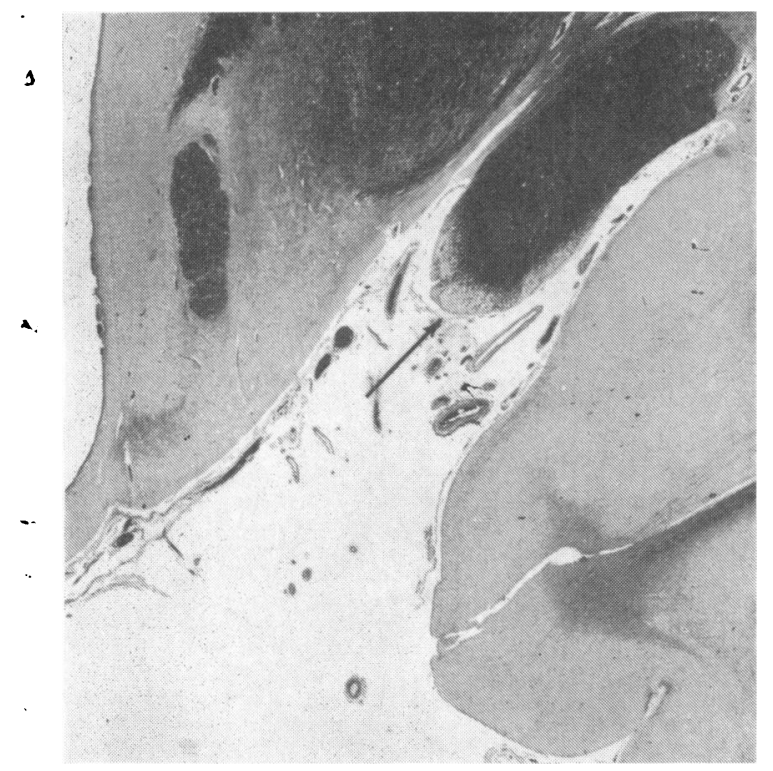

FIG. 4. Superficial destruction of the exposed surface of the optic tract, without changes in adjacent temporal cortex and tuber cinereum. Bacsich's stain, $\times 6$. Arrow indicates the optic tract.

of the patient. Other findings of interest were widespread metastatic carcinoma and two courses of radiotherapy. Neither of these factors is likely to have played a role in the production of the lesions.

The precise classification of the surface lesions poses some difficulty. The selective involvement of the white matter suggests a demyelinating process. Histologically, however, there was destruction of axons and cellular elements in the areas of myelin loss. Hence, the designation 'superficial or marginal demyelination' is improper, and the term 'superficial destruction of white matter' seems more fitting.

The lesions in our case resemble those found as complications of spinal anaesthesia with cocaine derivatives (Spielmeyer, 1908, 1909; Klose and Vogt, 1909; Bodechtel, 1928; Brock, Bell, and Davison, 1936; Russell, 1937; Guttman and Pardee, 1944; Courville, 1955), after magnesium sulphate injection (Guttman and Wolf, 1944), or the accidental spinal injection of a detergent (Winkelman, 1952). According to Pentschew (1958), such lesions may be classified as: (1) peripheral demyelination; (2) axonal reaction in the anterior horn motor neurones; (3) massive necrosis of the cord; (4) changes due to chronic, productive arachnitis. Only the first type of lesions is applicable to our case, although there was no evidence of the introduction of any of the substances mentioned above. Antibiotics were given intravenously or per os, except for an irrigation of the sella turcica with bacitracin solution during the revision. There is no known previous report of comparable histological changes in the CNS due to local application of antibiotics. Previous observations suggest that the use of skeletal muscle for closing the fistula is without adverse effects on the CNS. The lesions in the present case differ in certain details from those described in the above cases. The case discussed by Brain (1937) and by Russell (1937), for example, had massive necrosis of the cord, with patchy peripheral demyelination in segments cranial to the necrosis. A pattern of patchy demyelination, in addition to slight diffuse marginal demyelination, was also found in the case of Brock et al. (1936). Damage to dorsal roots was found in several cases, demonstrated either directly or by the ensuing degeneration of the dorsal tracts (Bodechtel, 1928; Guttman and Pardee, 1944; Winkelman, 1952). The latter two cases also showed focal loss of cells in the anterior horns. The marginal demyelination was mild and did not form a sharply delineated layer in the cases of Guttman and Pardee (1944), Winkelman (1952), and Courville (1955), and was shown to extend into the ventral median fissures in the case of Guttman and Wolf (1944). Even the case of Bodechtel (1928), in which the loss of fibres was quite marked, did not show the sharp delineation of the lesions found in our material.

Spielmeyer $(1908,1909)$ and Klose and Vogt (1909) produced degeneration of superficial fibres by intraspinal application of cocaine derivatives in dogs, monkeys, and rabbits; the cords of these animals also showed chromatolysis of motor neurones and damage to dorsal roots with degeneration of dorsal tracts. One of the illustrations by Spielmeyer clearly shows the superficial zone of damage extending into the ventral median fissure, but this observation is not emphasized in the text. Lesions following intraspinal injection of phenol (Berry and Olszewski, 1963) showed a pattern of damage reverse to that in our case: selective damage was found in the peripheral portion of roots, whereas the central portion and surface of cord were intact.

In conclusion, peripheral demyelination after spinal anaesthesia often shows additional damage in the grey matter and in roots, and only few cases on record are comparable in intensity with the changes found in the present case. None of the cases shows extension of the lesions above the cervical level.

Surface lesions produced by barbotage (cerebrospinal fluid pumping consisting of aspiration and forcible reinjection of cerebrospinal fluid) in cats (Bunge and Settlage, 1957; Bunge et al., 1960; 
Koenig, Bunge, and Bunge, 1962) were strikingly similar to our case. Common gross and histological features were the sharp delineation of the lesions from the intact tissue, their strict orientation to the surface and relatively uniform depth, the involvement of the medulla oblongata, the relative sparing of roots including the dorsal root entrance zones, and the failure of the lesions to extend along the ventral median fissure of the cord.

Another similarity with the experimental lesions lies in the clinical history of our case, in which the repeated, large drainings of cerebrospinal fluid constitute a manipulation similar to cerebrospinal fluid pumping, however without any reinjection. The drainings were probably more forceful and complete than in normal lumbar puncture since the $5 \mathrm{~mm}$ fistula in the floor of the sella must have accelerated the speed of outflow. Cases with a clinical history of cerebrospinal fluid fistula with repeated lumbar punctures were examined before, but no similar pathological findings were observed. This discrepancy may be explained by the fact that only about $50 \%$ of the animals subjected to CSF pumping by Bunge and Settlage (1957) developed lesions, due to differences unknown.

The present case is unique in that an experimental model producing comparable lesions was described before such changes were noted in human material. Yet, the pathogenesis remains obscure. Bunge and Settlage (1957) consider compression of the cord surface by arachnoid fluid pockets as a possible pathogenetic mechanism; as an alternative, they assume that pumping may render cerebrospinal fluid toxic to surface structures of the cord. Unfortunately, these assumptions offer little specific help in understanding the experimental model or our case, although a common denominator appears to be present.

\section{SUMMARY}

Necropsy findings are described in a case in which a thin layer with nearly complete destruction of white matter was found at the surface of the spinal cord, medulla oblongata, pons, optic nerve, and tract. The lesions were restricted to white matter. There was relative sparing of the nerve roots beyond the glia-Schwann-cell border. No evidence of the introduction of toxic substances into the cerebrospinal fluid was found.

The lesions in this case resemble those produced experimentally by cerebrospinal fluid pumping bye Bunge and Settlage (1957); the similarity is enhance by the fact that the present case had a cerebrospinal 0 fluid fistula in the sella and received numerous therapeutic drainings of cerebrospinal fluid.

\section{REFERENCES}

Bacsich, P., and Stibbe, E. P. (1937). A simple Weigert technique onf paraffin sections of CNS material. J. Anat., 72, 163-164.

Berry, K., and Olszewski, J. (1963). Pathology of intrathecal phenof injection in man. Neurology (Minneap.), 13, 152-154.

Bodechtel, G. (1928). Befunde am Zentralnervensystem bef? Spätnarkosetodesfällen und bei Todesfällen nach Lumbað anaesthesie. Ztschr. f.d. ges. Neurol. u. Psychiat., 117, 366-4239

Brain, W. R. (1937). In Discussion on the neurological sequelae of spinal anesthesia. Proc. roy. Soc. Med., 30, 1024-1025.

Brock, S. Bell, A., and Davison, C. (1936). Nervous complication following spinal anesthesia. A clinical study of seven cases, with tissue study in one instance. J. Amer. med. Ass., 106, 441-447.

Bunge, P., Bunge, M. B., and Ris, H. (1960). Electron microscopic study of demyelination in an experimentally induced lesion in adult cat spinal cord. J. biophys. biochem. Cytol., 7, 685-696.

Bunge, R. P., and Settlage, H. (1957). Neurological lesions in cates following cerebrospinal fluid manipulation. J. Neuropat $h$ U Exp. Neurol., 16, 471-491.

Courville, C. B. (1955). Untoward effects of spinal anesthesia on thy

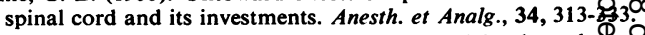

Guttman, S. A., and Pardee, I. (1944). Spinal cord level syndrome following intrathecal ammonium sulfate and procaine hydo chloride. A case report with autopsy findings. Anesthesiolazy 5, 347-353.

_-, and Wolf, A. (1944). Spinal cord level syndrome following intrathecal administration of magnesium sulfate for taboti crisis. Report of a case with autopsy. Arch. Neurol. Psyc 西a for (Chic.), 52, 135-139.

Klose, H., and Vogt, H. (1909). Experimentelle Untersuchungerfin Spinalanalgesie. Mitt. Grenzgeb. Med. Chir., 19, 737-805?

Koenig, H., Bunge, M. B., and Bunge, R. P. (1962). Nucleic acid and protein metabolism in white matter. Observations during experimental demyelination and remyelination; a histoo chemical and autoradiographic study of spinal cord of the adult cat. Arch. Neurol. (Chic.), 6, 177-193.

Pentschew, A. (1958). Zwischenfälle bei Lumbalanaesthesie und intralumbaler Medikation, pp. 2294-2304. In Handbuch deQ Speziellen Pathologischen Anatomie und Histologie. Vog XIII/2. Springer-Verlag, Berlin.

Russell, D. (1937). Myelomalacia following spinal anaesthesiō (pathological report), in Discussion on the neurologica sequelae of spinal anesthesia. Proc. roy. Soc. Med., 30, 10251030.

Spielmeyer, W. (1908). Veränderungen des Nervensystems nach Stovain anästhesie. Münch. med. Wschr., 55, 1629-1634.

(1909). Pseudosystemerkrankungen des Rückenmarkes nach Stovain-anästhesie. Neurol. Zentbl., 28, 69-80.

Winkelman, N. W. (1952). Neurological symptoms following acciō dental intraspinal detergent injection. Neurology (Minneap. 2, 284-291. 